\title{
A HAUSSMANN-CLARK-OCONE FORMULA FOR FUNCTIONALS OF DIFFUSION PROCESSES WITH LIPSCHITZ COEFFICIENTS
}

\author{
KHALED BAHLALI ${ }^{1}$ \\ Université de Toulon-Var, UFR Sciences \\ B.P. 132, 83957 La Garde Cedex, France \\ CPT, CNRS Luminy, Case 907 \\ 13288 Marseille Cedex 9, France \\ E-mail: bahlali@univ-tln.fr \\ BRAHIM MEZERDI ${ }^{2}$ \\ Université de Biskra \\ Département de Mathématiques \\ B.P. 145 Biskra Algérie \\ E-mail: bmezerdi@yahoo.fr \\ YOUSSEF OUKNINE ${ }^{1}$ \\ Université Cadi Ayyad \\ Département de Mathématiques, Faculté des Sciences Semlalia \\ B.P. S2390 Marrakech, Maroc \\ E-mail: ouknine@ucam.ac.ma
}

(Received April, 2001; Revised February, 2002)

\begin{abstract}
We establish a martingale representation formula for functionals of diffusion processes with Lipschitz coefficients, as stochastic integrals with respect to the Brownian motion.

Key words: Stochastic Integral, Stochastic Differential Equation, Haussmann-Clark-Ocone Formula.

AMS subject classifications: $60 \mathrm{H} 15,60 \mathrm{H} 10$.
\end{abstract}

\section{Introduction}

Let $\left(\Omega, \mathcal{F}, \mathcal{F}_{t}, P\right)$ be a filtered probability space satisfying the usual conditions on which a standard Brownian motion $\left\{B_{t} ; 0 \leq t \leq 1\right\}$ is defined; $\left(\mathcal{F}_{t}\right)_{0 \leq t \leq 1}$ is the natural filtration generated by $\left(B_{t}\right)$ and augmented with $P$-null sets. It is well known $[9,13]$ that, if $M_{t}$ is a $\mathcal{F}_{t}$-square integrable martingale, then there exists an $\mathcal{F}_{t}$-adapted process $H_{t}$ satisfying,

\footnotetext{
${ }^{1}$ Partially supported by CMIFM, AI No MA/01/02.

${ }^{2}$ Partially supported by French-Algerian Cooperation, PICS444.
} 


$$
E\left[\int_{0}^{1}\left|H_{t}\right|^{2} d t\right]<+\infty \text { such that } M_{t}=E\left[M_{0}\right]+\int_{0}^{t} H_{t} d B_{t} \text {. }
$$

If the martingale $M_{t}$ is given by the conditional expectation of some Brownian functional $M_{t}=E\left[L(B) / \mathcal{F}_{t}\right]$, where $L$ is a Frêchet differentiable functional defined on $\mathcal{C}([0,1])$ then Clark [4] has given an explicit representation of the process $H$, namely $H_{t}=E\left[\lambda^{L}((t, 1], B) / \mathcal{F}_{t}\right]$ where, $\lambda^{L}(d u, B)$ denotes the signed measure associated with the Frêchet derivative of $L(B)$. He also uses this formula to solve a stochastic nonlinear filtering problem. Haussmann [6] has extended this integral formula to functionals of Ito processes with smooth causal coefficients

$$
d X=b(t, X) d t+\sigma(t, X) d B
$$

and established the following integral representation

$$
L(X)=\int_{0}^{1} E\left\{\int_{(t, 1]} \lambda^{L}(d u, X) \Phi(u) \Phi^{-1}(t) / \mathcal{F}_{t}\right\} \sigma(t, X) d B_{t}
$$

where $\Phi(u)$ denotes the unique solution of the equation of first variation associated to $X$. The proof is based on Cauchy-Maruyama approximation. The last formula has been applied to stochastic control problems [8]. Later in [7], the author gave another proof of this result based on Girsanov's theorem. Note also that this formula played a key role in Bismut's version of Malliavin calculus [1]. Ocone [12] recovered the Haussmann-Clark formula by using Malliavin calculus techniques, and extended it to the class of functionals $L$ which are weakly $H$-differentiable. See Davis [5] for a proof using potential theory techniques.

The common assumption in all previous works is the differentiability of the coefficients $b, \sigma$ with respect to the state variable. This assumption allows one to show that $X_{t}^{x}$ admits a derivative with respect to initial condition, at least in the $L^{2}$ sense. In this paper we drop this hypothesis. We suppose that $b, \sigma$ are only Lipschitz continuous in the state variable. We establish an integral representation formula in which $\Phi(u)$ is defined by means of generalized derivatives of the coefficients $b, \sigma$. This is done in the nondegenerate, as well as in the degenerate case. The main idea in the proof is to show that even when the coefficients are merely Lipschitz, it is possible to associate to the diffusion process $X_{t}^{x}$ a unique linearized version defined as the distributional derivative of $X_{t}^{x}$ with respect to the initial condition. In the case where the matrix $\sigma(t, x) \sigma^{*}(t, x)$ is nondegenerate, our proof is carried out by using an approximation procedure and Krylov's inequality. Roughly speaking, this inequality says that the law of the random variable $X_{t}^{x}$ is absolutely continuous with respect to Lebesgue measure. This property allows us to define a unique linearized version of the stochastic differential equation (2.3). That is, if we choose two versions of the generalized derivatives of $b$ and $\sigma$, then the corresponding transition matrices are equal.

The method performed in the previous case is no longer valid in the degenerate case, and the sort of derivative (with respect to the initial condition) defined will have no sense. In this case, we make use of a result of Bouleau and Hirsch [2,3] to define a generalized derivative of the process defined on an enlarged probability space. The idea is to consider a slightly different stochastic differential equation defined on an enlarged probability space, where the initial condition $x$ will be taken as a random element. This allows us to perform operations outside negligible sets (in $x$ ), which are not possible for the initial equation. The method is inspired from result [2,3], where the authors proved an absolute continuity result, extending the well known Malliavin calculus methods. 


\section{Assumptions and Preliminaries}

Let $(\Omega, \mathcal{F}, P)$ be a probability space on which is defined a $d$-dimensional Brownian motion $\left(B_{t}\right)$, and let $\mathcal{F}_{t}$ be the $\sigma$-algebra generated by $\left\{B_{s} ; 0 \leq s \leq t\right\}$ completed with $P$-null sets of $\mathcal{F}$. Let $\mathcal{C}[0, T]$ be the space of $\mathbb{R}^{d}$-valued continuous functions defined on $[0, T]$, equipped with the topology of uniform convergence. $\mathcal{B}(\mathcal{C})$ denotes its Borel $\sigma$-field.

Let $L: \mathcal{C}[0, T] \rightarrow \mathbb{R}$ be a functional satisfying,

$L$ is a measurable, polynomially growing, Frêchet differentiable functional in $C[0, T]$, with differential $L^{p}(x) y$.

$$
L^{\prime}: C[0, T] \rightarrow C^{*}[0, T] \text { is continuous and grows polynomially. }
$$

According to the Riesz theorem, there exists a right continuous function $\mu_{x}(t)=$ $\left(\mu_{x}^{1}(t), \mu_{x}^{2}(t), \ldots, \mu_{x}^{d}(t)\right)$ of bounded variation such that,

$$
L^{\prime}(x) y=\int_{0}^{1} d \mu_{x}(t) y(t)
$$

Consider the stochastic differential equation

$$
\left\{\begin{array}{c}
d X_{t}^{x}=b\left(t, X_{t}^{x}\right) d t+\sigma\left(t, X_{t}^{x}\right) d W_{t} \\
X_{0}^{x}=x
\end{array}\right.
$$

whose coefficients are Borel measurable functions satisfying $\exists M>0$ such that $\forall(t, x, y) \in \mathbb{R}_{+} \times \mathbb{R}^{d} \times \mathbb{R}^{d}$

$$
\begin{gathered}
|\sigma(t, x)-\sigma(t, y)|+|b(t, x)-b(t, y)| \leq M|x-y| \\
|\sigma(t, x)|+|b(t, x)| \leq M(1+|x|) .
\end{gathered}
$$

The assumptions (2.4) and (2.5) guarantee the existence and uniqueness of a strong solution for equation (2.3), such that for any $p>0, E\left[\sup _{t \leq T}\left|x_{t}\right|^{p}\right]<+\infty$. Since the functions $b, \sigma^{j}$ (the $j$ th column of the matrix $\sigma$ ) are Lipschitz continuous in the state variable, then according to the Rademacher theorem they are differentiable almost everywhere in the sense of Lebesgue measure. Let us denote by $b_{x}, \sigma_{x}^{j}$ any Borel measurable functions such that

$$
\begin{aligned}
& \frac{\partial b}{\partial x}=b_{x}(t, x) \quad d x \text { a.e. } \\
& \frac{\partial \sigma^{j}}{\partial x}=\sigma_{x}^{j}(t, x) \quad d x \text { a.e. }
\end{aligned}
$$

It is clear that these generalized derivatives are bounded by the Lipschitz constant $M$. Let us recall Krylov's inequality which will play a key role in the next section. 
Theorem 2.1: (Krylov [10]) Let $\left(\Omega, \mathcal{F}, \mathcal{F}_{t}, P\right)$ be a filtered probability space, $\left(B_{t}\right)$ a ddimensional Brownian motion, $b: \Omega \times \mathbb{R}_{+} \rightarrow \mathbb{R}^{d}, \sigma: \Omega \times \mathbb{R}_{+} \rightarrow \mathbb{R}^{d} \otimes \mathbb{R}^{d}$ bounded adapted processes such that:

$$
\exists \lambda>0, \forall \xi \in \mathbb{R}^{d} \xi^{*} \sigma \sigma^{*} \xi \geq \lambda|\xi|^{2} .
$$

Let $X_{t}=x+\int_{0}^{t} b(t, \omega) d t+\int_{0}^{t} \sigma(t, \omega) d B_{t}$ be an Ito process. Then for every Borel function $f: \mathbb{R}_{+} \times \mathbb{R}^{d} \rightarrow \mathbb{R}$ with support in $[0, T] \times B(0, M)$ the following inequality holds:

$$
E\left[\int_{0}^{T}\left|f\left(t, x_{t}\right)\right| d t\right] \leq K\left[\int_{B(0, M)}^{T}|f(t, x)|^{d+1} d t d x\right]^{1 / d+1}
$$

where $K$ is a constant and $B(0, M)$ stands for the ball of center 0 and radius $M$.

\section{The Nondegenerate Case}

Assume that the hypothesis in the last section hold and suppose that the diffusion matrix $\sigma(t, x)$ satisfies the uniform ellipticity condition:

$$
\exists \lambda>0, \forall \xi \in \mathbb{R}^{d}, \xi^{*} \sigma(t, x) \xi \geq \lambda|\xi|^{2}
$$

Let us denote by $\Phi(s, t)$ the solution of the following first variation equation associated with $X_{t}^{x}$

$$
\left\{\begin{array}{c}
d \Phi(s, t)=b_{x}\left(s, X_{t}^{x}\right) \Phi(s, t) d s+\sum_{1 \leq j \leq d} \sigma_{x}^{j}\left(s, X_{t}^{x}\right) \Phi(s, t) d W_{s}^{j} \\
\Phi(t, t)=I_{d}
\end{array}\right.
$$

where $b_{x}, \sigma_{x}^{j}$ are generalized derivatives of $b, \sigma^{j}$.

The main result of this section is the following:

Theorem 3.1: Under assumptions (2.4), (2.5) and (3.1), it holds that

$$
L\left(X^{x}\right)=\int_{0}^{1} E\left[\lambda(t) / \mathcal{F}_{t}\right] \sigma\left(t, X_{t}^{x}\right) d W_{t}+E\left[L\left(X_{t}^{x}\right)\right]
$$

where $\lambda(t)=\int_{t}^{1} \mu\left(d s, X^{x}\right) \Phi(s, t)$.

Proof: Let $\varphi$ be a $\mathcal{C}^{\infty}$ nonnegative function defined on $\mathbb{R}^{d}$, with support in the unit ball such that $\int \varphi(y) d y=1$. For $n \in \mathbb{N}^{*}$, define the following smooth functions

$$
\begin{gathered}
b^{n}(t, x)=n^{d} \int b(t, x-y) \varphi(n y) d y \\
\sigma^{j, n}(t, x)=n^{d} \int \sigma^{j}(t, x-y) \varphi(n y) d y .
\end{gathered}
$$


It is a classical fact that the functions $b^{n}(t, x), \sigma^{j, n}(t, x)$ are Borel measurable functions and $M$-Lipschitz continuous in $x$ such that:

$$
\begin{gathered}
\left|b^{n}(t, x)-b(t, x)\right|+\left|\sigma^{j, n}(t, x)-\sigma^{j}(t, x)\right| \leq C / n=\varepsilon_{n} \\
\sigma^{j, n}(t, x), b^{n}(t, x) \text { are } C^{1} \text { functions in } x \text {, and for each } t \in[0, T], \\
\lim _{n \rightarrow+\infty} \sigma_{x}^{j, n}(t, x)=\sigma_{x}^{j}(t, x) \operatorname{and}_{n \rightarrow \infty} \lim _{x} b_{x}^{n}(t, x)=b_{x}(t, x) d x \text {-a.e. }
\end{gathered}
$$

Consider the sequence of stochastic differential equations

$$
\left\{\begin{array}{c}
d X_{t}^{n}=b^{n}\left(t, X_{t}^{n}\right) d t+\sigma^{n}\left(t, X_{t}^{n}\right) d B W_{t} \\
X_{0}^{n}=x
\end{array}\right.
$$

The approximating coefficients $b^{n}(t, x), \sigma^{j, n}(t, x)$ satisfying the conditions (2.4), (2.5), moreover they are smooth in $x$ with bounded derivatives. Therefore we are in a position to apply the Haussmann representation formula for the functional $L\left(X^{n}\right)$ (see [4]) which gives

$$
L\left(X^{n}\right)=\int_{0}^{1} E\left[\lambda^{n}(t) / \mathcal{F}_{t}\right] \sigma^{n}\left(t, X_{t}^{n}\right) d W_{t}+E\left[L\left(X_{t}^{n}\right)\right]
$$

where $\lambda^{n}(t)=\int_{t}^{1} \mu\left(d s, X^{n}\right) \Phi^{n}(s, t)$ and

$$
\left\{\begin{array}{c}
d \Phi^{n}(s, t)=b_{x}^{n}\left(s, x_{s}^{n}\right) \Phi^{n}(s, t) d s+\sum_{1 \leq j \leq d} \sigma_{x}^{j, n}\left(s, X_{s}^{n}\right) \Phi^{n}(s, t) d W_{s}^{j} \\
\Phi^{n}(t, t)=I_{d}
\end{array}\right.
$$

The continuity and the polynomial growth condition satisfied by $L$ guarantee the convergence of $L\left(X^{n}\right)$ and $E\left[L\left(X^{n}\right)\right]$ to $L(X)$ and $E[L(X)]$ respectively. Condition (2.2) ensures the convergence of $\mu\left(d s, x^{n}\right)$ to $\mu(d s, x)$ in $C^{*}[0, T]$. It remains to show that $\Phi^{n}(s, t)$ converges uniformly in probability to $\Phi(s, t)$, and $\Phi(s, t)$ does not depend on Borel versions of the generalized derivatives of $b$ and $\sigma^{j}(j=1,2, \ldots, d)$. This will be the subject of the next two lemmas.

Lemma 3.2: $\Phi(s, t)$ is well defined and does not depend on Borel versions of the generalized derivatives of $b$ and $\sigma$ up to $P$ a.s. equality.

Proof: Let $b_{x}^{1}, b_{x}^{2}$ be two Borel versions of the derivative of $b$ at $x$. That is, for each $t \in[0, T], b_{x}^{1}(t, x)=b_{x}^{2}(t, x) d x$ a.e. Let $\sigma_{x}^{j, 1}, \sigma_{x}^{j, 2}$ defined similarly.

Denote by $\Phi^{1}(s, t)$ (resp. $\left.\Phi^{2}(s, t)\right)$ the solution of (3.2) corresponding to $b_{x}^{1}, \sigma_{x}^{j, 1}$ (resp. $\left.b_{x}^{2}, \sigma_{x}^{j, 2}\right)$. Then by Gronwall's inequality, we have

$$
E \quad\left[\sup _{t \leq s \leq T}\left|\Phi^{1}(s, t)-\Phi^{2}(s, t)\right|^{2}\right] \leq K\left\{E\left[\int_{0}^{T}\left|b_{x}^{1}\left(t, X_{t}^{x}\right)-b_{x}^{2}\left(t, X_{t}^{x}\right)\right|^{2} d t\right]\right.
$$




$$
\begin{gathered}
\left.\operatorname{li}_{1 \leq j \leq d} E\left[\int_{0}^{T}\left|\sigma_{x}^{j, 1}\left(t, X_{t}^{x}\right)-\sigma_{x}^{j, 2}\left(t, X_{t}^{x}\right)\right|{ }^{2} d t\right]\right\} \\
=K\left\{I_{1}+I_{2}\right\} .
\end{gathered}
$$

Since for $p>0, \quad E\left[\sup _{t \leq T}\left|X_{t}^{x}\right|^{p}\right]<+\infty$, we have $\lim _{M \rightarrow+\infty} P\left[\sup _{t \leq T}\left|X_{t}^{x}\right|>\right.$ $M]=0$.

Therefore without loss of generality, we may suppose that $b_{x}^{1}, b_{x}^{2}, \sigma_{x}^{j, 1}, \sigma_{x}^{j, 2}$ have compact support $[0, T] \times B(0, M)$. By applying Krylov's inequality (thanks to condition (3.1)), we obtain

$$
I_{1} \leq N\left\|b_{x}^{2}-b_{x}^{2}\right\|_{d+1, M}=0
$$

The fact that $I_{2}=0$ can be proved using similar arguments.

Equation (2.5) satisfies the Lipschitz conditions, due to the boundedness of the coefficients $b_{x}$ and $\sigma_{x}^{j}$. Hence it has a unique strong solution.

Lemma 3.3: Let $\Phi^{n}(s, t)$ (resp. $\Phi(s, t)$ ) be the solution of (3.6) (resp. (3.2)). Then the following estimate holds

$$
E \quad\left[\sup _{s \leq t \leq T}\left|\Phi^{n}(s, t)-\Phi(s, t)\right|^{2}\right] n \rightarrow+\infty \infty^{0 .}
$$

Proof: Using the Burkholder-Davis-Gundy, Schwarz and Gronwall inequalities, we obtain

$$
\begin{aligned}
& E\left[\sup _{s \leq t \leq T} \mid\right.\left.\Phi^{n}(s, t)-\left.\Phi(s, t)\right|^{2}\right] \leq M E \quad\left[\sup _{s \leq t \leq T}\left|\Phi^{n}(s, t)\right|^{4}\right]^{1 / 2} \\
& \times\left\{E\left[\int_{0}^{T}\left|b_{x}^{n}\left(t, X_{t}^{n}\right)-b_{x}\left(t, X_{t}^{x}\right)\right|{ }^{4} d t\right]^{1 / 2}\right. \\
&\left.+\sum_{1 \leq j \leq d} E\left[\int_{0}^{T}\left|\sigma_{x}^{j, n}\left(t, X_{t}^{n}\right)-\sigma_{x}^{j}\left(t, X_{t}^{x}\right)\right|^{4} d t\right]^{1 / 2}\right\} .
\end{aligned}
$$

Since the coefficients in the linear stochastic differential equation (3.6) are bounded, $\Phi^{n}(s, t)$ is uniformly (in $t$ ) bounded in $L^{p}$ for each $p \geq 1$. Therefore, the first term in the right hand side is finite. To derive the desired result, it is sufficient to prove the following: 


$$
\begin{gathered}
I_{1}^{n}=E\left[\int_{0}^{T}\left|b_{x}^{n}\left(t, X_{t}^{n}\right)-b_{x}\left(t, X_{t}^{x}\right)\right|^{4} d t\right] \rightarrow 0 \text { as } n \rightarrow+\infty \\
I_{1}^{j, n}=E\left[\int_{0}^{T}\left|\sigma_{x}^{j, n}\left(t, X_{t}^{n}\right)-\sigma_{x}^{j}\left(t, X_{t}^{x}\right)\right|^{4} d t\right] \rightarrow 0 \text { as } n \rightarrow+\infty,
\end{gathered}
$$

where $j=1,2, \ldots, d$.

Let us prove the first one. Let $n_{0} \geq 1$ be a fixed integer, then it holds that:

$$
\begin{gathered}
\lim _{n \rightarrow+\infty} I_{1}^{n} \leq \limsup _{n \rightarrow+\infty} M_{1}\left\{E\left[\int_{0}^{T} b_{x}^{n}\left(t, X_{t}^{n}\right)-b_{x}^{n_{0}}\left(t, X_{t}^{n}\right) \mid{ }^{4} d t\right]\right. \\
+E\left[\int_{0}^{T}\left|b_{x}^{n_{0}}\left(t, X_{t}^{n}\right)-b_{x}^{n_{0}}\left(t, X_{t}^{x}\right)\right|{ }^{4} d t\right] \\
\left.+E\left[\int_{0}^{T}\left|b_{x}^{n_{0}}\left(t, X_{t}^{x}\right)-b_{x}\left(t, X_{t}^{x}\right)\right|{ }^{4} d t\right]\right\} \\
=M_{1}\left(J_{1}^{n}+J_{2}^{n}+J_{3}^{n}\right) .
\end{gathered}
$$

Let $w(t, x)$ be a continuous function such that $w(t, x)=0$ if $t^{2}+x^{2} \geq 1$ and $w(0,0)=1$. Then for $M>0$, we have

$$
\begin{gathered}
\limsup _{n \rightarrow+\infty} J_{1}^{n} \leq N M_{1}\left\{E\left[\int_{0}^{T}\left(1-w\left(\frac{t}{M}, \frac{x_{t}}{M}\right)\right) d t\right]\right. \\
\left.+\limsup _{n \rightarrow+\infty} E\left[\int_{0}^{T} w\left(\frac{t}{M}, \frac{x_{t}}{M}\right)\left|b_{x}^{n}\left(t, x_{t}^{n}\right)-b_{x}^{n_{0}}\left(t, x_{t}^{n}\right)\right|{ }^{4} d t\right]\right\} .
\end{gathered}
$$

Applying Krlov's inequality we obtain

$$
\limsup _{n \rightarrow+\infty} J_{1}^{n} \leq N M_{1}\left\{\left[E\left(\int_{0}^{T}\left(1-w\left(\frac{t}{M}, \frac{x_{t}}{M}\right)\right) d t\right]\right.\right.
$$




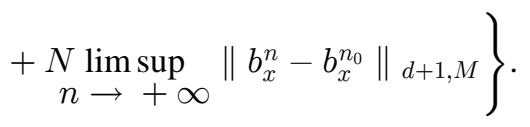

Note that we have used the fact that the diffusion matrix $\sigma^{n}(t, x)$ satisfies the nondegeneracy condition with the same constant $\lambda$ as $\sigma(t, x)$. According to the fact that $b_{x}^{n}$ converges to $b_{x}, d x$-a.e., the last expression in the right hand side tends to 0 as $n_{0}$ tends to $+\infty$. Next, letting $M$ go to $+\infty$ and using properties of the function $w(t, x)$ we conclude that $\lim \sup _{n \rightarrow+\infty} J_{3}^{n}=0$. We use the continuity of $b_{x}^{n_{0}}$ in $x$ and the convergence in probability (uniformly in $t$ ) of $X_{t}^{n}$ to $X_{t}^{x}$ to see that $\lim _{n \rightarrow+\infty} b_{x}^{n_{0}}\left(t, X_{t}^{n}\right)=b_{x}^{n_{0}}\left(t, X_{t}^{x}\right)$ in

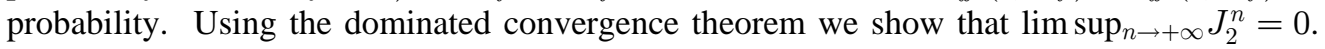
Hence $\lim _{n \rightarrow+\infty} I_{I}^{n}=0$.

By the same arguments, one can prove that for each $j=1,2, \ldots, d, \lim _{n \rightarrow \infty} I_{2}^{j, n}=0$, which completes the proof.

Remark 3.4: Suppose that for each $t \geq 0$, the law of $X_{t}^{x}$ is absolutely continuous with respect to Lebesgue measure. Assume moreover that the family $\left\{p^{n}(t, y), n \geq 1\right\}$ of densities of the approximating processes $X_{t}^{n}$ is equi-integrable. Then formula (3.3) remains valid.

\section{The Degenerate Case}

Throughout this section, the reference probability space is the usual Wiener space. We assume that the coefficients $b, \sigma$ and the functional $L$ satisfy the hypotheses of the last section except condition (3.1) on the uniform ellipticity of $\sigma(t, x) \sigma^{*}(t, x)$.

Let us recall some preliminaries and notations which will be applied in this section to establish the Haussmann-Clark representation formula. See [2, 3] for details and proofs.

Let $\Omega=\mathcal{C}_{0}\left(\mathbb{R}_{+}, \mathbb{R}^{d}\right)$ be the space of continuous functions $\omega$ such that $\omega(0)=0$, endowed with the topology of uniform convergence on compact subsets of $\mathbb{R}_{+}$. Let $\mathcal{F}$ be the Borel $\sigma$ field over $\Omega, P$ be the Wiener measure on $(\Omega, \mathcal{F})$ and $\left(\mathcal{F}_{t}\right)_{t \geq 0}$, the filtration of coordinates augmented with $P$-null sets of $\mathcal{F}$.

Let us define the canonical process $B_{t}(\omega)=\omega(t)$, for all $t \geq 0$. $\left(\Omega, \mathcal{F},\left(\mathcal{F}_{t}\right)_{t \geq 0}, P, B_{t}\right)$ is then a Brownian motion.

Let $h$ be a continuous positive function on $\mathbb{R}^{d}$ such that $\int h(x) d x=1$ and $\int|x|{ }^{2} h(x) d x<+\infty$. Define the space $D=\left\{f \in L^{2}(h d x)\right.$, such that $\frac{\partial f}{\partial x_{j}} \in L^{2}(h d x)$, $j=1, \ldots, d\}$, where $\frac{\partial f}{\partial x_{j}}$ denotes the derivative in the sense of distributions.

Equipped with the norm

$$
\|f\|_{D}=\left[\int f^{2} h d x+\sum_{1 \leq j \leq d} \int\left(\frac{\partial f}{\partial x_{j}}\right)^{2} h d x\right]^{1 / 2} .
$$

$D$ is a Hilbert space, which is a classical Dirichlet space $[2,3]$. Note also that $D$ is a subset of the Sobolev space $H_{\text {loc }}^{1}\left(\mathbb{R}^{d}\right)$.

Let $\widetilde{\Omega}=\mathbb{R}^{d} \times \Omega, \widetilde{\mathcal{F}}$ the Borel $\sigma$-field over $\widetilde{\Omega}$ and $\widetilde{P}=h d x \otimes P$.

Let $\widetilde{B}_{t}(x, \omega)=B_{t}(\omega), \widetilde{\mathcal{F}}_{t}$ be the natural filtration of $\widetilde{B}_{t}$ augmented with $\widetilde{P}$-negligible sets. It is clear that $\left(\widetilde{\Omega}, \widetilde{\mathcal{F}},\left(\widetilde{\mathcal{F}}_{t}\right)_{t \geq 0}, \widetilde{P}, \widetilde{B}_{t}\right)$ is a Brownian motion.

Let us define the Hilbert space $\widetilde{D}$ which is a general Dirichlet space (see [3]) 


$$
\widetilde{D}=\left\{\begin{array}{c}
u: \widetilde{\Omega} \rightarrow \mathbb{R}, \exists \widetilde{u}: \widetilde{\Omega} \rightarrow \mathbb{R} \text { Borel measurable such that } u=\widetilde{u}, \widetilde{P} \text {-a.e. and } \\
\forall(x, \omega) \in \widetilde{\Omega}, t \rightarrow u\left(x+t e_{i}, \omega\right) \text { is locally absolutely continuous. }
\end{array}\right\}
$$

Let $\widetilde{X}_{t}$ be the process defined on the enlarged space $\left(\widetilde{\Omega}, \widetilde{\mathcal{F}},\left(\widetilde{\mathcal{F}}_{t}\right)_{t>0}, \widetilde{P}, \widetilde{B}_{t}\right)$, which is the solution of the following SDE

$$
\left\{\begin{array}{c}
d \widetilde{X}_{t}=b\left(t, \widetilde{X}_{t}\right) d t+\sigma\left(t, \widetilde{X}_{t}\right) d \widetilde{B}_{t} \\
\widetilde{X}_{0}=x
\end{array}\right.
$$

Since the coefficients $b, \sigma$ are Lipschitz continuous and grow at most linearly, (4.1) has a unique, continuous, $\widetilde{\mathcal{F}}_{t}$-adapted solution. Note that equations (2.3) and (4.1) are almost the same except that uniqueness for (4.1) is slightly weaker. One can easily prove that the uniqueness implies that for each $t \geq 0, \widetilde{X}_{t}=X_{t}, \widetilde{P}$-a.s.

Theorem 4.1: (Bouleau-Hirsch [2, 3]) For P-almost every $\omega$

(i) For all $t \geq 0, X_{t}(\omega) \in D^{d} \subset\left(H_{l o c}^{1}\left(\mathbb{R}^{d}\right)\right)^{d}$.

(ii) There exists a $\widetilde{\mathcal{F}}_{t}$-adapted, $G L_{d}(\mathbb{R})$-valued continuous process $\left(\widetilde{\Phi}_{t}\right)$ such that for P-almost every $\omega$ :

$$
\forall t \geq 0, \frac{\partial}{\partial x}\left(X_{t}^{x}(\omega)\right)=\widetilde{\Phi}_{t}(x, \omega), d x \text {-a.e. }
$$

where $\frac{\partial}{\partial x}$ denotes the derivative in the sense of distributions.

Remark 4.2: As a consequence of the last theorem, the image measure of $\widetilde{P}$ by the map $\widetilde{X}_{t}$ is absolutely continuous with respect to Lebesgue measure.

Lemma 4.3: The distributional derivative $\widetilde{\Phi}_{t}$ is the unique solution of the linear stochastic differential equation

$$
\left\{\begin{array}{c}
d \widetilde{\Phi}(s, t)=b_{x}\left(s, \widetilde{X}_{s}\right) \widetilde{\Phi}(s, t) d s+\sum_{1 \leq j \leq d} \sigma_{x}^{j}\left(s, \widetilde{X}_{s}\right) \widetilde{\Phi}(s, t) d \widetilde{B}_{s}^{j} \\
\widetilde{\Phi}(t, t)=I_{d}
\end{array}\right.
$$

where $b_{x}$ and $\sigma_{x}^{j}$ are versions of the almost everywhere derivatives of $b$ and $\sigma^{j}$.

Proof: Since the image measure of $\widetilde{P}$ by the map $\widetilde{X}_{t}$ is absolutely continuous with respect to Lebesgue measure, $\widetilde{\Phi}(s, t)$ is well defined and does not depend on the versions of the Borel derivatives $b_{x}, \sigma_{x}^{j}$. Moreover, since the coefficients $b_{x}\left(s, \widetilde{X}_{s}\right)$ and $\sigma_{x}^{j}\left(s, \widetilde{X}_{s}\right)$ are bounded, equation (4.2) satisfy Lipschitz conditions and has a unique $\widetilde{\mathcal{F}}_{t}$-adapted continuous solution. The fact that $\widetilde{\Phi}(s, t)$ satisfies equation (4.2) is based on the absolute continuity of the law of $\widetilde{X}_{t}$ and on the approximation of the coefficients $b$ and $\sigma$ by smooth ones. See [2] for details.

The main result of this section is the following.

Theorem 4.4: Assume that the coefficients $b, \sigma$ and the functional $L$ satisfy hypotheses (2.1)-(2.5). Then the following martingale representation formula holds: 


$$
L(\widetilde{X})=\int_{0}^{1} E\left[\widetilde{\lambda}(t) / \widetilde{\mathcal{F}}_{t}\right] \sigma\left(t, \widetilde{X}_{t}\right) d \widetilde{B}_{t}+\widetilde{E}[L(\widetilde{X})] \quad \widetilde{P} \text {-a.s. }
$$

where $\widetilde{\lambda}(t)=\int_{t}^{1} \mu(d s, \widetilde{X}) \widetilde{\Phi}(s, t)$ and $\widetilde{\Phi}(s, t)$ is the distributional derivative of $X_{t}^{x}(\omega)$ with respect to $x$.

The proof of Theorem 4.4 is based on the next two lemmas.

Lemma 4.5: Let $b^{k}, \sigma^{j, k}$ be the smooth functions defined in the last section. Let the processes $\widetilde{Y}_{t}^{k}, \widetilde{\Psi}^{k}(s, t)$ be defined on $\left(\widetilde{\Omega}, \widetilde{\mathcal{F}},\left(\widetilde{\mathcal{F}}_{t}\right)_{t \geq 0}, \widetilde{P}, \widetilde{B}_{t}\right)$ by the stochastic differentials

$$
\begin{gathered}
\left\{\begin{array}{c}
d \widetilde{Y}_{t}^{k}=b^{k}\left(t, \widetilde{X}_{t}\right) d t+\sigma^{k}\left(t, \widetilde{X}_{t}\right) d \widetilde{B}_{t} \\
\widetilde{Y}_{0}^{k}=x
\end{array}\right. \\
\left\{\begin{array}{c}
d \widetilde{\Psi}^{k}(s, t)=b_{x}^{k}\left(s, \widetilde{X}_{s}\right) \widetilde{\Phi}(s, t) d s+\sum_{1 \leq j \leq d} \sigma_{x}^{j, k}\left(s, \widetilde{X}_{s}\right) \widetilde{\Phi}(s, t) d \widetilde{B}_{s}^{j} \\
\widetilde{\Psi}^{k}(s, t)=I_{d} .
\end{array}\right.
\end{gathered}
$$

Then,

and

$$
\lim _{k \rightarrow+\infty} E\left[\sup _{s \leq t \leq T}\left|\widetilde{Y}_{t}^{k}-\widetilde{X}_{t}\right|^{2}\right]=0
$$

$$
\lim _{k \rightarrow+\infty} E\left[\sup _{s \leq t \leq T}\left|\widetilde{\Psi}^{k}(s, t)-\widetilde{\Phi}(s, t)\right|^{2}\right]=0
$$

Proof: To prove that $\widetilde{Y}_{t}^{k} \rightarrow \widetilde{X}_{t}$ we use the fact that for each $t \geq 0, b^{k}(t, x)$, (resp. $\sigma^{k}(t, x)$ ) converges uniformly to $b(t, x)$ (resp. $\left.\sigma(t, x)\right)$ and apply the dominated convergence theorem. Applying the standard arguments of stochastic differential equations yields

$$
\begin{aligned}
E\left[\sup _{s \leq t \leq T}\left|\widetilde{\Psi}_{k}(s, t)-\widetilde{\Phi}(s, t)\right|^{2}\right] \leq M E\left[\sup _{s \leq t \leq T}|\widetilde{\Phi}(s, t)|^{4}\right]^{1 / 2} \\
\\
\times\left\{E\left[\int_{0}^{T}\left|b_{x}^{k}\left(t, \widetilde{X}_{t}\right)-b_{x}\left(t, \widetilde{X}_{t}\right)\right|^{4} d t\right]^{1 / 2}\right.
\end{aligned}
$$




$$
\left.+\sum_{1 \leq j \leq d} E\left[\int_{0}^{T}\left|\sigma_{x}^{j, k}\left(t, \widetilde{X}_{t}\right)-\sigma_{x}^{j}\left(t, \widetilde{X}_{t}\right)\right|{ }^{4} d t\right]^{1 / 2}\right\}
$$

Clearly $E\left[\sup _{s \leq t \leq T}|\widetilde{\Phi}(s, t)|^{4}\right]<+\infty$. Let us prove that

$$
E \quad\left[\int_{0}^{T}\left|b_{x}^{k}\left(t, \widetilde{X}_{t}\right)-b_{x}\left(t, \widetilde{X}_{t}\right)\right|{ }^{4} d t\right] \text { converges to } 0 \text { as } k \rightarrow+\infty \text {. }
$$

Let $\widetilde{p}(t, y)$ be the density of $\widetilde{X}_{t}$ with respect to Lebesgue measure. Then

$$
E\left[\int_{0}^{T}\left|b_{x}^{k}\left(t, \widetilde{X}_{t}\right)-b_{x}\left(t, \widetilde{X}_{t}\right)\right|{ }^{4} d t\right]=\int_{0}^{T} \int_{\mathbb{R}^{d}}\left|b_{x}^{k}(t, y)-b_{x}(t, y)\right|{ }^{4} \widetilde{p}(t, y) d t d y .
$$

Since $b_{x}^{k}$ and $b_{x}$ are bounded by the Lipschitz constant and $\left(4.4^{\prime}\right), b_{x}^{k} \rightarrow b_{x}$, a.e., follows from the dominate convergence theorem. Using the same technique, we prove that for each $j=1, \ldots, d$,

$$
\lim _{k \rightarrow+\infty} E\left[\int_{0}^{T}\left|\sigma_{x}^{j, k}\left(t, \widetilde{X}_{t}\right)-\sigma_{x}^{j}\left(t, \widetilde{X}_{t}\right)\right|{ }^{4} d t\right]=0
$$

Lemma 4.6: Let $\widetilde{X}_{t}^{k}$ and $\widetilde{\Phi}^{k}(s, t)$ be the unique solutions of the following stochastic differential equations:

$$
\begin{gathered}
\left\{\begin{array}{c}
d \widetilde{X}_{k t}=b^{k}\left(t, \widetilde{X}_{t}^{k}\right) d t+\sigma^{k}\left(t, \widetilde{X}_{t}^{k}\right) d \widetilde{B}_{t} \\
\widetilde{Y}_{0}^{k}=x
\end{array}\right. \\
\left\{\begin{array}{c}
d \widetilde{\Phi}^{k}(s, t)=b_{x}^{k}\left(s, \widetilde{X}_{t}^{k}\right) \widetilde{\Phi}^{k}(s, t) d s+\sum_{1 \leq j \leq d} \sigma_{x}^{j, k}\left(s, \widetilde{X}_{t}^{k}\right) \widetilde{\Phi}^{k}(s, t) d \widetilde{B}_{s}^{j} \\
\widetilde{\Phi}^{k}(s, t)=I_{d} .
\end{array}\right.
\end{gathered}
$$

Then as $k \rightarrow+\infty$

$$
E \quad\left[\sup _{s \leq t \leq T}\left|\widetilde{X}_{t}^{k}-\widetilde{X}_{t}\right|^{2}\right] \rightarrow 0 \text { and } E \quad\left[\sup _{s \leq t \leq T}\left|\widetilde{\Phi}^{k}(s, t)-\widetilde{\Phi}(s, t)\right|^{2}\right] \rightarrow 0 .
$$


Proof: Similar to the proof of the previous lemma.

Proof of Theorem 4.4: Since $b^{k}$ and $\sigma^{k}$ are smooth functions,

$$
L\left(X^{n}\right)=\int_{0}^{1} E\left[\lambda^{n}(t) / \mathcal{F}_{t}^{W}\right] \sigma^{n}\left(t, X_{t}^{n}\right) d W_{t}+E\left[L\left(X^{n}(\cdot)\right)\right]
$$

where $\lambda^{n}(t)=\int_{t}^{1} \mu\left(d s, X^{n}\right) \Phi^{n}(s, t), X^{n}$ and $\Phi^{n}(s, t)$ are given by equations (3.4) and (3.6). Since $X^{n}=\widetilde{X}_{n}$ and $\Phi^{n}(s, t)=\widetilde{\Phi}^{n}(s, t), \widetilde{P}$-a.s.,

$$
\left.L\left(\widetilde{X}^{n}\right)=\int_{0}^{1} E\left[\widetilde{\lambda}^{n}\right) / \widetilde{\mathcal{F}}_{t}\right] \sigma^{n}\left(t, \widetilde{X}_{t}^{n}\right) d \widetilde{B}_{t}+\widetilde{E}\left[L\left(\widetilde{X}_{t}(t)\right)\right]
$$

where

$$
\widetilde{\lambda}^{n}(t)=\int_{t}^{1} \mu\left(d s, \widetilde{X}^{n}\right) \widetilde{\Phi}^{n}(s, t)
$$

According to Lemmas 4.5 and 4.6 and the continuity of $\mu(d s, \cdot)$, one can pass to the limit in the last formula and obtain the desired result. Note that we have proved the convergence in $L^{2}$, but in fact this convergence is valid in $L^{p}$ for each $p \geq 2$.

Corollary 4.7: Assume that for each $t \geq 0$, the law of $X_{t}^{x}$ is absolutely continuous with respect to Lebesgue measure. Then the Hausmann-Clark formula given in Theorem 4.4 holds on the probability space $\left(\Omega, \mathcal{F}, \mathcal{F}_{t}, P\right)$.

Proof: Consider the sequence of processes

$$
\begin{gathered}
\left\{\begin{array}{c}
d X_{t}^{n}=b^{n}\left(t, X_{t}^{x}\right) d t+\sigma^{n}\left(t, X_{t}^{x}\right) d B_{t} \\
X_{0}^{n}=x
\end{array}\right. \\
\left\{\begin{array}{c}
d \Phi^{n}(s, t)=b_{x}^{n}\left(s, X_{t}^{x}\right) \Phi(s, t) d s+\sum_{1 \leq j \leq d} \sigma_{x}^{j, n}\left(s, X_{t}^{x}\right) \Phi(s, t) d B_{s}^{j} \\
\Phi^{n}(t, t)=I_{d}
\end{array}\right.
\end{gathered}
$$

where $\Phi(s, t)$ is the unique solution of the linear SDE

$$
\left\{\begin{array}{c}
d \Phi(s, t)=b_{x}\left(s, X_{t}^{x}\right) \Phi(s, t) d s+\sum_{\substack{1 \leq j \leq d\\
}} \sigma_{x}^{j}\left(s, X_{t}^{x}\right) \Phi(s, t) d B_{s}^{j} \\
\Phi(t, t)=I_{d} .
\end{array}\right.
$$


Note that since the law of $X_{t}^{x}$ is absolutely continuous with respect to Lebesgue measure, $\Phi(s, t)$ is well defined (the proof is performed as in Lemma 3.2). Now apply the HausmannOcone formula for $L\left(X^{n}\right)$ (see [11]) to obtain

$$
L\left(X^{n}\right)=E\left[L\left(X^{n}\right)\right]+\int_{0}^{1} E\left[D_{t} L\left(X^{n}\right) / \mathcal{F}_{t}\right] d W_{t}
$$

where $D_{t} L\left(X^{n}\right)$ denotes the Malliavin derivative of $L\left(X^{n}\right)$, given by

$$
D_{t} L\left(X^{n}\right)=\int_{t}^{1} \mu\left(d s, X^{n}\right) D_{t}\left(X_{s}^{n}\right)
$$

and $D_{t}\left(X_{s}^{n}\right)=\Phi^{n}(s, t) \sigma^{n}\left(t, X_{t}^{x}\right)$.

We conclude by observing that $E\left[\sup _{s \leq t \leq T}\left|X_{t}^{n}-X_{t}\right|^{2}\right] \rightarrow 0$ and

$$
E \quad\left[\sup _{s \leq t \leq T}\left|\Phi^{n}(s, t)-\Phi(s, t)\right|^{2}\right] \rightarrow 0 \text { as } k \rightarrow+\infty \text {. }
$$

Remark 4.8: In particular, the absolute continuity assumption is satisfied in the one dimensional case and the coefficients $b, \sigma$ are time independent such that $\sigma(x) \neq 0$ for some initial condition.

\section{References}

[1] Bismut, J.M., Martingales, the Malliavin calculus and hypoellipticity under general Hörmander's condition, Z. Wahrshein 56 (1981), 469-505.

[2] Bouleau, N. and Hirsch, F., Dirichlet Forms and Analysis on Wiener Space, De Gruyter Studies in Math 14, Berlin 1991.

[3] Bouleau, N. and Hirsch, F., On the Derivability with Respect to the Initial Data of the Solution of a Stochastic Differential Equation with Lipschitz Coefficients, Sém. Théorie du Potenital 9, Lect. Notes in Math., Springer-Verlag, Berlin 1989.

[4] Clark, J.M.C., The representation of functionals of Brownian motion by stochastic integrals, Ann. Math. Stat. 41 (1970), 1282-1295.

[5] Davis, M.H.A., Functionals of diffusion processes as stochastic integrals, Math. Proc. Cambridge Phil. Soc. 87 (1980), 157-166.

[6] Hausmann, U.G., Functionals of Ito processes as stochastic integrals, SIAM J. Cont. Optim. 16 (1978), 252-269.

[7] Hausmann, U.G., On the integral representation of functionals of Ito processes, Stochastics 3 (1979), 17-27.

[8] Hausmann, U.G., A Stochastic Maximum Principle for Optimal Control of Diffusions, Longman Scientific and Technical, Essex, UK 1986.

[9] Kallianpur, G., Stochastic Filtering Theory, Springer-Verlag, Berlin 1980. 
[10] Krylov, N.V., Controlled Diffusion Processes, Springer-Verlag, Berlin 1980.

[11] Nualart, D., The Malliavin Calculus and Related Topics, Springer-Verlag, Berlin 1995.

[12] Ocone, D., Malliavin's calculus and stochastic integral representation of functionals of diffusion processes, Stochastics 12 (1984), 161-185.

[13] Ustunel, S., An Introduction to Analysis on Wiener Space, Lect. Notes in Math. 1610, Springer-Verlag, Berlin 1995. 


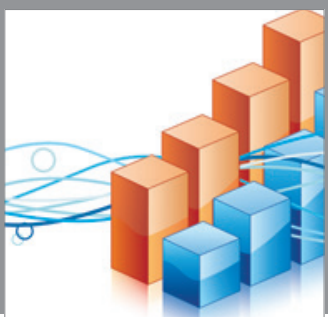

Advances in

Operations Research

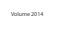

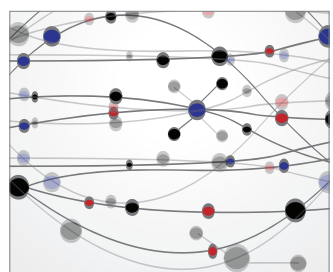

\section{The Scientific} World Journal
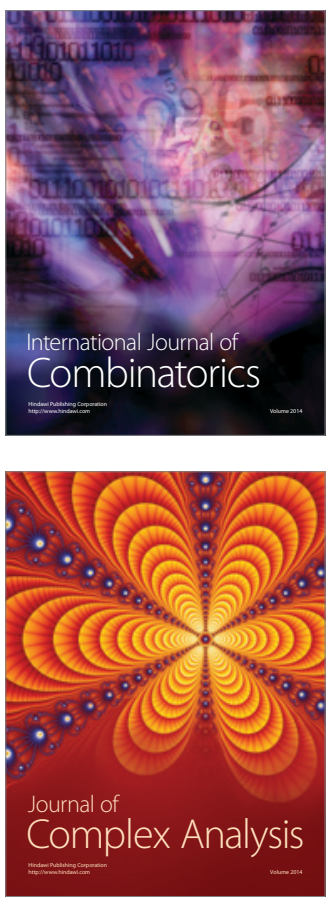

International Journal of

Mathematics and

Mathematical

Sciences
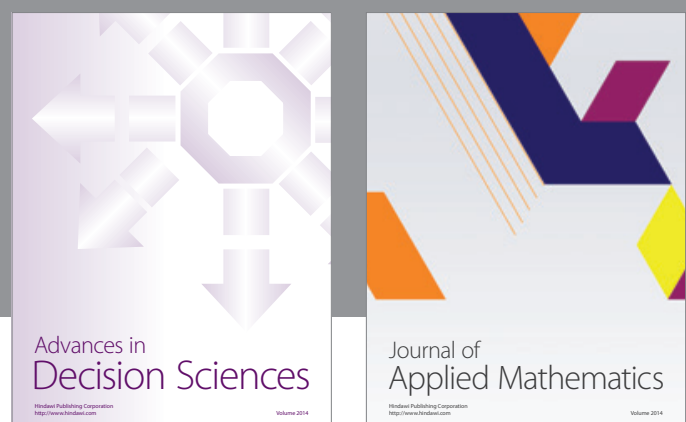

Journal of

Applied Mathematics
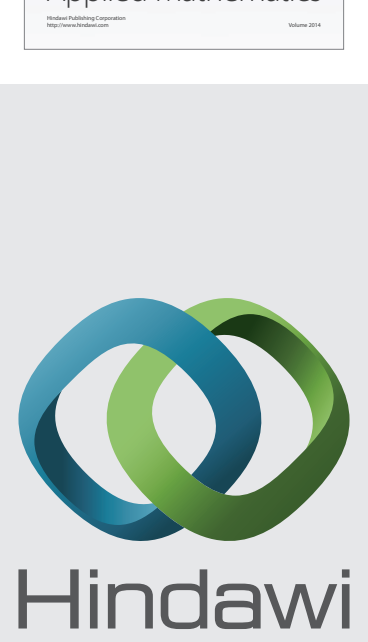

Submit your manuscripts at http://www.hindawi.com
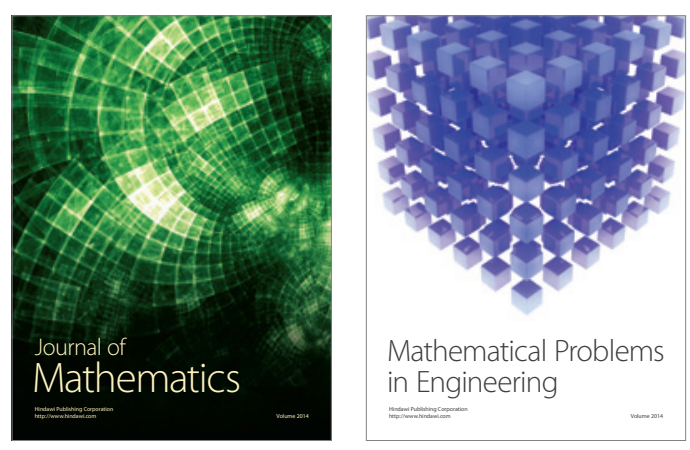

Mathematical Problems in Engineering
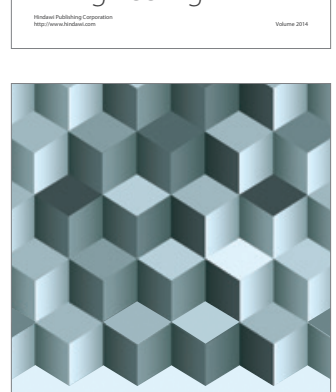

Journal of

Function Spaces
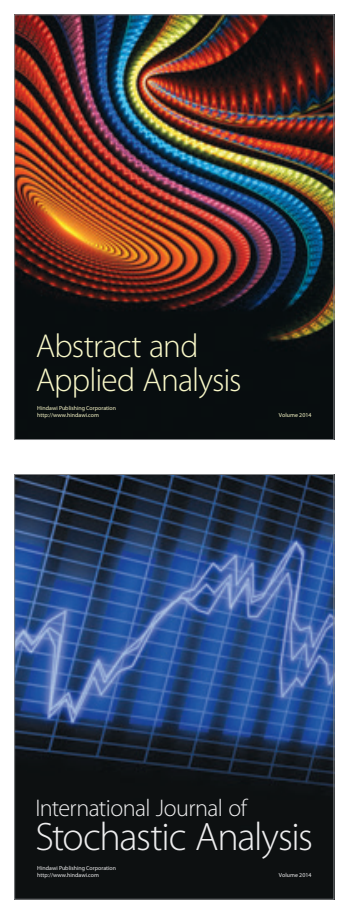

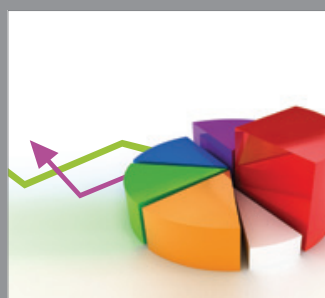

ournal of

Probability and Statistics

Promensencen
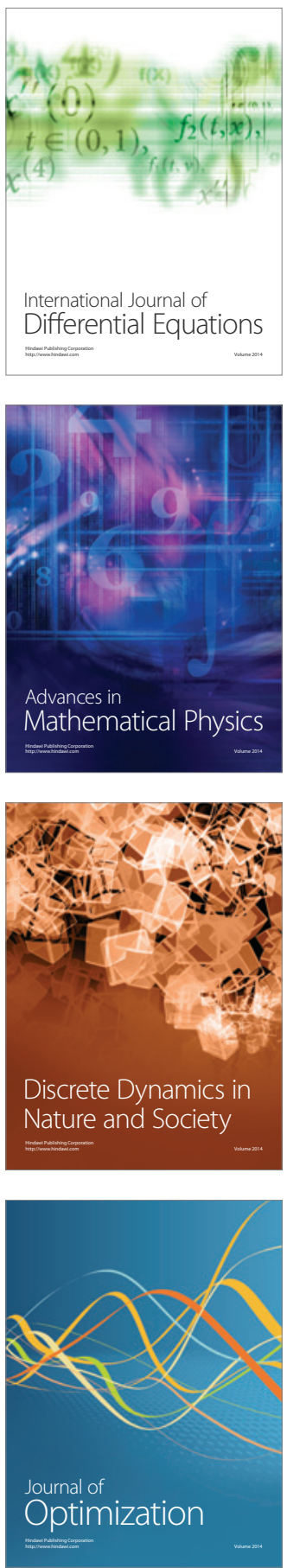